\title{
Host Immunity Biomarkers of Neonatal Sepsis: A Prospective, Multicentre Study in Sub Saharan Africa
}

\section{Sem Ezinmegnon}

Institute of Integrative Biology of the Cell

Marine Mommert

1. Open Innovation \& Partnerships Lyon Sud (OI\&P)

Francois Bartolo

Soladis Statistical Inc

\section{Gino Agbota}

Institut de Recherche pour le Développement

\section{Sossou Darius}

Institut de Recherche Clinique du Benin

\section{Valérie Briand}

Institut de Recherche pour le Développement

\section{Marceline d'Almeida}

Centre National Hospitalier et Universitaire Hubert Koutoukou MAGA

\section{Maroufou Jules Alao}

Mother and Child University and Hospital Center (CHUMEL)

Ida Dossou-Dagba

Calavi Hospital

Achille Massougbodji

Institut de Recherche Clinique du Benin

\section{Ulrik Lausten-Thomsen}

Assistance Publique - Hôpitaux de Paris

\section{Alexandre Pachot}

Open Innovation \& Partnerships Lyon Sud (OI\&P)

\section{Laurence Vachot}

Open Innovation \& Partnerships Lyon Sud (OI\&P)

Javier Yugueros-Marcos

Open Innovation \& Partnerships Lyon Sud (OI\&P)

\section{Karen Brengel-Pesce}

Open Innovation \& Partnerships Lyon Sud (OI\&P)

\section{Nadine Fievet}


Institut de Recherche pour le Développement

Pierre Tissieres ( $\sim$ pierre.tissieres@aphp.fr)

Institute of Integrative Biology of the Cell

\section{Research Article}

Keywords: sepsis, neonate, biomarkers, diagnosis, procalcitonin, CD74, human leukocyte antigen, HLA, CX3CR1, IP-10, IL-6, IL-10, low-income countries, biofire, mortality

Posted Date: July 15th, 2021

DOl: https://doi.org/10.21203/rs.3.rs-561585/v2

License: (c) (7) This work is licensed under a Creative Commons Attribution 4.0 International License. Read Full License 


\section{Abstract}

Few biomarkers for sepsis diagnosis are commonly used in neonatal sepsis. Whilst host response is increasingly recognized in sepsis pathogenesis and prognosis, there is a need for evaluating the accuracy of new biomarkers targeting host response in regions where sepsis burden is high and medico-economic resources are scarce. The objective of the study is to evaluate diagnostic and prognostic accuracy of biomarkers of neonatal sepsis in Sub Saharan Africa. This prospective multicentre study included newborn infants delivered in the Abomey-Calavi region in South Benin and their follow-up from birth to 3 months of age. Accuracy of transcriptional (CD74, CX3CR1), proteic (PCT, IL-6, IL-10, IP-10) biomarkers and clinical characteristics to diagnose and prognose neonatal sepsis were measured. At delivery, cord blood from all consecutive newborns were sampled and analysed, and infants were followed for a 12 weeks' period. Five hundred and eighty-one newborns were enrolled. One hundred and seventy-two newborns developed neonatal sepsis (29.6\%) and death occurred in forty-nine infants (8.4\%). Among all tested biomarkers and clinical criteria, CD74/IP-10 ratio showed the best accuracy for neonatal sepsis diagnosis, while PCT accuracy was low. Among biomarkers and clinical criteria studied, only CD74 and PCT were independently associated with mortality, with $C D 74$ showing an elevated predictive accuracy. These results suggest that cord blood PCT had a low accuracy for diagnosing early onset neonatal sepsis in Sub Saharan African neonates, while CD74/IP-10 ratio had the best diagnostic accuracy. $C D 74$ expression at birth had the best accuracy in prognosing sepsis mortality.

Trial registration: Clinicaltrial.gov registration number: NCT03780712. Registered 19 December 2018. Retrospectively registered.

\section{Introduction}

Neonatal sepsis remains a major societal and economic burden in low- and middle-income countries. The Institute for Health Metrics and Evaluation estimates that in 2017, 200,000 newborns deaths occurred for every 2 million cases of sepsis in sub-Saharan Africa (SSA) ${ }^{1}$. Early diagnosis of newborns with sepsis can reduce mortality and morbidity rates ${ }^{2}$. Unfortunately, the limited specificity of clinical signs, the lack of appropriate biological testing and the high cost of treatment in low-income countries hamper most initiatives to reduce neonatal and pediatric mortality ${ }^{3}$. Difficulty in diagnosing sepsis in neonates is related to the conjunction of multiple variables, such as maternal health, pregnancy conditions, gestational age at delivery, their immune functionality, timing of infection and pathogens detection, as well as socio-economic environments and access to healthcare.

At birth, the immune defences go through rapid development and strengthen during the first years of life ${ }^{4}$. Studies have shown that the increased risk of early and late neonatal infections observed in both term and preterm infants are related to impaired innate immune function ${ }^{5-7}$. This innate immaturity is characterized by altered cytokines production, decreased function of antigen-presenting cells and has many similarities to the immunosuppression phenotypes observed in critically ill adults and septic patients ${ }^{8,9}$. In addition to factors known to be associated with neonatal infection ${ }^{10,11}$, frequent parasitic 
infections in SSA, such as malaria, toxoplasmosis and schistosomiasis, affect foetal and perinatal growth and development ${ }^{12,13}$. In low-income countries, the specific example of gestational malaria (GM) shows that sequestration and cytoadhesion of infected red blood cells in the placental intercellular space lead to local inflammatory responses, altered placental function and subsequently promote intrauterine growth retardation and prematurity ${ }^{14,15}$. The in utero impact of GM on immunity in early childhood is recognized and may explain the altered immune response to vaccines and the increased risk of infection $16,17$.

Blood culture remains the gold standard for the diagnosis of neonatal sepsis, but results are delayed and have low sensitivity due to maternal antibiotic treatment, small sample volumes, and low bacteremia 18,19 . Other tests routinely used for the diagnosis of neonatal sepsis, such as C-reactive protein (CRP) and blood count (total and differential white blood cell counts, absolute and immature neutrophil counts, and the ratio of immature to total neutrophils), are respectively non-specific and non-sensitive ${ }^{20-23}$. Procalcitonin (PCT) has been shown to be an excellent marker for severe bacterial infection in newborn 24-26. However during the first 48 hours following birth, high PCT values may reflect a state of stress rather than the presence of a neonatal infection ${ }^{25,27}$. PCT therefore suffers, in some cases, of a lack of sensitivity for diagnosis of neonatal sepsis. Several studies have shown that some pro-inflammatory (IL6, IP-10) and anti-inflammatory (IL-10) cytokines are acute phase biomarkers that can be used for the diagnosis of bacterial infection in neonatal. Levels of IL-6, IL-10 and IP-10 are significantly higher in neonatal sepsis than in healthy controls ${ }^{28-30}$. IL- 6 and IP-10 are associated with inflammation in newborn ${ }^{31,32}$, while IL-10 is associated with immunosuppression and mortality in adult septic patients ${ }^{33}$. However, those cytokine profiles may be influenced by the gestational age and time of onset of infection in newborn ${ }^{29,34}$. Based on a microarray study, a panel of genes has been identified in critically ill septic patients whose expression on peripheral blood could effectively help stratifying patients at increased risk of secondary infection and/or death ${ }^{35,36}$. Among these genes, CD74 and CX3CR1 were shown to be associated with mortality and/or the occurrence of secondary infections ${ }^{37-39}$. Low expression of $C X 3 C R 1$ is associated with mortality and immunosuppression in adult patients with septic shock ${ }^{37-39}$. Similarly, low expression of $C D 74$ is associated with mortality in adult patients with septic shock while increasing expression of $C D 74$ in the first days of intensive care is associated with the occurrence of secondary infections ${ }^{38,40}$. There are no data on the accuracy of $C D 74$ and $C X 3 C R 1$ for the prognosis or diagnosis of neonatal sepsis, which hamper current use of these biomarkers in neonates and children $22,23,27,41$.

Few studies have accessed neonatal sepsis epidemiology in malaria endemic SSA and characterized it with extensive diagnostic workup including systematic blood cultures, pathogen molecular diagnosis and patients biomarkers of sepsis and immune status ${ }^{42}$. In this study, we evaluated the accuracy of transcriptional (CX3CR1, CD74) and proteic (IL6, IL10, IP-10, PCT) biomarkers and their association with clinics and maternal risk factors to diagnose and prognose neonatal sepsis and obtained the reference range of those biomarkers during the first three months of life. 


\section{Method}

\section{Study design and participants}

Participants were delivered in two sub-urban health centres (sub-urban arm) and three urban University hospitals (hospital arm) in the Abomey-Calavi, Sô-Ava and Cotonou districts in the South Benin region where malaria is hyper-endemic ${ }^{43}$. In both arms, only infants born from mother living in the AbomeyCalavi district were recruited to facilitate the follow-up and minimize effect of geographical origins. In the sub-urban arm, that includes only normal geatation with low-risk delivery (no maternal risk factors for infection), all consecutive births were included, whereas in the hospital arm only newborns born from mothers with maternal-foetal risk factors for infection (prematurity, prolonged rupture of the membrane, maternal fever) were included. In both arms, the exclusion criteria included maternal HIV positive status, major congenital malformation and refusal of consent. All children from both arms were followed clinically on a bi-monthly base during the first 3 months of life. The follow-up consisted of scheduled home visits and unscheduled emergency visits if the infant was ill. The study protocol was approved by the Comité d'Ethique de la Recherche - Institut des Sciences Biomédicales Appliquées (CER-ISBA 85-5). Written informed consent was obtained from parents. All methods were performed in accordance with the relevant guidelines and regulations.

\section{Exposures and neonatal sepsis definition}

The exposure were occurrence of gestational malaria (GM). GM was defined as a malaria infection during pregnancy or at delivery. For women in the suburban arm, malaria screening was performed at each scheduled prenatal visit using a thick blood smear. Mothers from the Hospital arm were screened only at the time of delivery. In this group, antenatal malaria was established on the basis of mother's anamnesis. For both study arms, placental blood smear and mother's peripheral blood smear were performed. The Lambaréné technique was used to quantify parasitaemia with a detection threshold of five parasites per microliter ${ }^{44}$.

Neonatal sepsis was suspected in neonates with more than two of the following criteria being present: neutrophil count $<7500 / \mathrm{mm} 3$ or $>14500 / \mathrm{mm} 3$, band form $>1500 / \mathrm{mm} 3$, immature/total neutrophils ratio $>0.16$, platelets count $<150000 / \mathrm{mm} 3$ and CRP $>10 \mathrm{mg} / \mathrm{L}$. Suspected neonatal sepsis was considered as clinical sepsis when the following clinical signs were associated: temperature irregularity; respiratory distress or apnoea; seizures, altered tonus, irritability or lethargy; vomiting, altered feeding pattern, ileus; skin perfusion alteration, haemodynamic signs (tachycardia, hypotension); hypoglycaemic/hyperglycaemic, hyperlactatemia or identification of focal infection such as soft tissue infection or conjunctivitis. All newborns with a clinical sepsis were subsequently adjudicated by one independent pediatrician (PT) and sorted into 'presumed sepsis' and 'definite clinical sepsis' grouped as "adjudicated sepsis". In discordant cases, a second independent pediatrician (ULT) performed the final adjudication with access, in addition to the full medical file review, to microbiological cultures results. Parallel to microbiological culture (BACT/ALERT ${ }^{\circledR}$ system), specific BioFire ${ }^{\circledR}$ FilmArray ${ }^{\circledR}$ panels 
(bioMerieux, Marcy-l'Etoile, France) were run for all positive blood cultures (Blood Culture Identification (BCID) panel), cerebrospinal fluids (meningitis/encephalitis panel), respiratory and gastrointestinal samples (Pneumonia and Gastro-Intestinal panels). All studied biomarkers were kept blinded for the adjudication.

\section{Biomarkers sampling}

At birth and at follow-up visits, the clinical examination data of the children were collected. Blood samples were obtained at birth, then at week (W)1, W4, W8 and W12. The study protocol has been described in detail elsewhere ${ }^{44}$. Sampling and analytical methods are presented in Supplementary Appendix 2

\section{Outcomes}

The primary outcome was the diagnosis of clinical neonatal sepsis, and secondary outcome was mortality within the first three months of life. Neonatal sepsis diagnosis was established by the local paediatrician based on the clinical examination of the child and initial laboratory workup including haemogram, C-reactive protein (CRP) and microbiological cultures (blood, cerebral fluids and urine). Neonatal sepsis that occurred within the first 72 hours following birth was considered as an early onset neonatal sepsis (EONS), and late onset (LONS) thereafter (for detailed algorithm for sepsis diagnosis, see published study protocol ${ }^{44}$ ).

\section{Statistical analysis}

An independent statistician (FB) (Soladis Inc. Lyon, France; https://www.soladis.com/ ) supported the statistical methodology and performed all analysis. Statistical analyses were performed using R software version 3.6.1. The variables were assessed for normality using Kolmogorov Smirnov test. Numbers and frequency were used for qualitative data and medians and IQR (inter-quartile range: [Q1-Q3]) for quantitative data. Qualitative variables were compared using the Chi-squared test (or Fisher's exact test for small expected numbers). The distribution of quantitative data was compared using Student's t-test (or the Mann-Whitney t-test when distribution was not normal or Welch test when homoscedasticity was rejected) if 2 groups were compared. If more than 2 groups, the distribution of quantitative data was compared using Anova test (or the Kruskal-Wallis test when distribution was not normal or when homoscedasticity was rejected).

To evaluate their diagnostic accuracy, data-driven analysis was performed. Selection of cut-off values or discrimination values defining the positive and negative test results were performed. Several methods for selecting optimal cut-off values in diagnostic tests are proposed in the literature depending on the underlying reason for this choice. Here, we selected a cut-off to have a sensitivity of 0.95 and maximize specificity. This choice of cut-off was the same in the rest of the publication ${ }^{45}$. CD74/IP-10 was the score corresponding to the division of CD74 gene expression level by IP-10 serum concentration. We used three datasets to test more complex models, either genes (noted CX3CR1 \& CD74), proteins (noted Protein 
biomarkers) or sets of biomarkers (noted All biomarkers). To avoid overfitting and to compare our models, we used random sampling which takes place within each class and must preserve the overall distribution of data by class. To do this, we created a distribution, repeated 200 times, of $75 / 25 \%$ of the data. This distribution was used to optimize hyperparameters with package caret. Then we compared the average AUC and select the best average AUC for each dataset between all models. AUC accuracy were compared using Bootstrap approach. A p value $<0.05$ was considered as significant. Statistical algorithms descriptions are displayed in Supplementary Appendix 2.

For comparison between clinical variables (VC) and biomarkers, we selected the clinical variables of interest following an expert opinion (PT) and corresponding to neonatal risk factors (eg. Gestational age, weight, maternal risk factors, multiple gestation, APGAR score) (Supplementary Table 1). In order to compare clinical and biomarker data, we transformed the data into the same referential to be able to compare them. We transformed the categorical clinical variables into a complete set of dummy variables 46. Different transformations were then applied to the data set. A Yeo-Johnson transform is a non-linear transformation that reduces skewness and approximates a normal law. We centred (subtracts the mean of the variable's data) and scaled data (divides the standard deviation).

For the establishment of heatmap, Partial Last Squares (PLS) Regression was used to compare the two datasets. This algorithm comes from the mixOmics package. Biomarkers were deflated with respect to the information extracted/modelled from the local regression on Clinical Variables. Consequently, the latent variables computed to predict Biomarkers from Clinical Variables are different from those computed to predict Clinical Variables from Biomarkers. One matrix Clustered Image Map (CIM) is a 2dimensional visualization with rows and/or columns reordered according to some hierarchical clustering method to identify interesting patterns. The CIM allows to visualize correlations between variables. Generated dendrograms from clustering were added to the left side and to the top of the image. The used clustering method for rows and columns is the complete linkage method and the used distance measure is the distance Euclidean. We showed only variables with co-variances greater than max(covariance)/2.

$\mathrm{N}$-integration and feature selection with Projection to Latent Structures models (PLS) with sparse Discriminant Analysis was used to compare the two datasets and outcome (Circosplot). This algorithm comes from the mixOmics package. The circos plot represents the correlations between variables of different types, represented on the side quadrants. We showed within and between connexions between blocks, expression levels of each variable according to each class. The circos plots were built based on a similarity matrix, extended to the case of multiple data sets. We showed only variables with co-variances greater than $\max ($ correlation)/2 [46].

\section{Results}

\section{Cohort description}


Between April 17, 2016 and March 12, 2018, a total of 581 newborns were enrolled, 420 (72.3\%) in the hospital arm and $161(27.7 \%)$ in the sub-urban arm. Patients' characteristics are displayed in Table 1. Median gestational age (GA) was 38.4 weeks (95\% confidence interval [Cl], 35.5 to 40 weeks), with a median birth weight of 2816 grams ( $95 \% \mathrm{Cl}, 2400$ to 3150 grams). One hundred and eighty-four (31.6\%) infants were born prematurely with thirty-four (5.8\%) being less than 32 weeks of GA. More preterm infants were delivered in the hospital arm than in the sub-urban arm $(41.2 \%$ vs $6.2 \%, p<0.0001)$ and had a lower birth weight ( 2700 grams vs 3006 grams, $p<0.001)$. Forty-five multiple gestations (40 twins, 5 triplicate) happened. Gestational malaria occurred in forty-three mothers (8.1\%), affecting 49 infants (8.4\%). Intermittent preventive treatment of malaria was performed in $470 / 531$ (88.5\%) of all mothers. According to the study design, main differences between arms were related to the perinatal risks with more premature infants and maternal risk factors of infection in the hospital arm. In total, 432/581 (74.3 $\%$ ) babies were born from mothers with maternal risk factors of infection (386/531, 72.7\%). One hundred and seventy-two infants (29.6\%) developed a clinical sepsis according to the definition. All occurred in the hospital arm. Among these, adjudication confirmed 168 cases (97.7\%) (Figure 1). All but five clinical sepsis were EONS. In the hospital arm, 307/420 infants were hospitalized following birth, the remaining with uneventful delivery and normal perinatal adaptation were discharged. Only one infant from the suburban arm was hospitalized for prenatally undiagnosed omphalocele. The median hospital stay was 4 days ( $95 \% \mathrm{Cl}, 2$ to 7 days). The global mortality was 49/581 (8.4\%) with a significant difference between hospital and sub-urban arms, respectively $11.4 \%(48 / 420)$ versus $0.62 \%(1 / 161)(p<0.001)$. Most death occurred in the first week of life (median 2 days) and were related to neonatal sepsis $(n=44)$ or prematurity complications $(n=5)$.

Identification of microorganisms in neonatal sepsis was performed using conventional bacterial culture followed by Biofire ${ }^{\circledR}$ FilmArray ${ }^{\circledR}$ multiplexing panel testing. Results are described in Table 2. A total of 155 hemocultures were sampled, of which positive cultures was obtained in 59/155 (38.1\%) samples. Standard microbial cultures identified 59 pathogens and 14 hemocultures showed multiple germs and were considered as contaminated. Fifty-six of the 155 hemocultures were positive using Biofire ${ }^{\circledR}$ FilmArray ${ }^{\circledR}(56 / 155,36.1 \%)$, in which 91 pathogens were identified. Gram-negative bacteria represented the majority of pathogens (52.5\% to $49.4 \%$ pending the microbiologic technique) with Enterobacter cloacae complex, Klebsiella pneumonia, Serratia marcescens and Staphylococcus spp. being the most frequent germs identified (Table 2). Additional Biofire ${ }^{\circledR}$ FilmArray ${ }^{\circledR}$ panels identified viral infections (including Norovirus GI/GII, Rotavirus A, Coronavirus OC43, human rhinovirus and Respiratory Syncytial Virus) in 23 samples with negative standard cultures. Out of the 29 cerebrospinal fluid samples, one was positive for Hemophilus infuenzae.

\section{Primary outcome: Sepsis diagnosis and prognosis accuracy}

Cord blood levels of studied biomarkers are displayed in Table 3. Procalcitonin level was significantly higher in infants developing neonatal sepsis compared to both sub-urban arm and hospital healthy infants (Figure 2A). A significant difference in cord blood from non-septic neonates between both arms 
was observed. Diagnosis accuracy of cord blood PCT was low with an AUC of 0.62 ( $95 \% \mathrm{Cl}, 0.56$ to 0.67 ) (Figure 2B). Procalcitonin value of $401.8 \mathrm{pg} / \mathrm{mL}$ had a sensitivity of $96 \%$ but a poor specificity of $6 \%$ for clinical sepsis. Similarly, individual markers or most combinations had a low accuracy for sepsis diagnosis, but $C D 74 / \mathrm{IP}-10$ ratio showed a significant better accuracy than PCT $(\mathrm{p}=0.0015), C D 74$ $(p<0.001)$ and all biomarkers model $(p=0.046)$, respectively with an AUC of $0.77,0.62,0.59$ and 0.68 . No significant difference in diagnostic accuracy between biomarkers and clinical criteria association was seen. $C D 74 /$ IP-10 ratio value of 0.44 (Youden index 0.21 ) had a sensitivity of $73 \%$ and specificity of $25 \%$ for clinical sepsis diagnosis. Correlation between biomarkers and clinical variables showed that CX3CR1, IP-10, IL-6 and IL-10 are poorly correlated (Figure 3A). However, CD74 and PCT were strongly correlated (correlation cut-off <-0.5 or $>0.5$ ) with premature rupture of membrane (PROM), maternal fever, Apgar score, heart and respiratory rate (Figure 3A). More specifically, CD74 and PCT were inversely correlated with Apgar score, maternal fever, and to a lower extend with gestational age (Figure 3B).

\section{Secondary outcome: Mortality}

At delivery, PCT, CD74, IL-6 and CX3CR1 levels were significantly different between survivors and non survivors (Figure 4A). Prognosis accuracy of $C D 74$ was elevated (AUC $0.78 ; 95 \% \mathrm{Cl}, 0.68$ to 0.87 ) and low for CX3CR1 (AUC $0.51 ; 95 \% \mathrm{Cl}, 0.40$ to 0.61 ) (Figure 4B). CX3CR1, PCT and IP-10 levels were significantly different in survivors from both arms, suggesting that clinical conditions (maternal risk factors, prematurity) may affect those biomarkers (Figure 4A). Out of studied biomarkers, only CD74 and PCT were associated with mortality in a partial least squares regression model. $C D 74$ was positively correlated with Apgar score, gestational age, height and weight (Figure 5). Interestingly models combining clinical criteria and biomarkers had elevated accuracy for mortality, but were statistically similar. Model associating CD74 expression, Apgar score at 1 minutes and abnormal amniotic fluid had an AUC of 0.86 $(95 \% \mathrm{Cl}, 0.79$ to 0.92$)$.

\section{Biomarkers reference range in the first 12 weeks of life}

In healthy newborns, all biomarkers but PCT had a significant lower value on cord blood than in the first week following birth (Figure 6). Whereas most biomarkers range did not vary between weeks 1 and 12 , $C D 74$ progressively increased during the first eight weeks of life. Following neonatal sepsis, at week 4 , $C D 74$ was significantly lower in septic than in healthy newborns. We did not observe any significant differences in biomarker profiles between newborns exposed or not to gestational malaria.

\section{Discussion}

This multicentre study performed in sub Saharan Africa showed that cord blood PCT has a low accuracy for diagnosing neonatal sepsis whilst $C D 74 /$ IP-10 ratio had the best diagnostic accuracy among tested biomarkers and clinical criteria. In addition, the study showed that $C D 74$ accuracy was elevated in prognosing mortality. The evaluation of clinical and biomarkers for neonatal sepsis diagnosis and 
prognosis in setting with high prevalence of sepsis and limited resources is bringing for the first time detailed assessment of potential biomarkers utility and establishment of reference values in neglected pediatric populations. As such, this study is a radical breach in biomarkers development paradigms by refocusing their evaluation in setting with high prevalence of neonatal sepsis. Several large studies, occurring in setting with high resources and low sepsis prevalence, have suggested that cord blood PCT may be a robust biomarker for neonatal sepsis diagnosis, reporting elevated sensitivity and specificity for cut-off values ranging between $0.6-0.7 \mathrm{ng} / \mathrm{mL}^{47-49}$. These encouraging data were not confirmed by all. Few series in extreme premature infants and in setting where EONS prevalence was higher showed lower sensitivity and specificity ranging between 48.7 to $69 \%$ and 68.6 to $70 \%$, respectively ${ }^{50,51}$. Our study confirms the inaccuracy of cord blood PCT in diagnosing neonatal sepsis. The lack of specificity that we observed may be related to the effect of maternal and neonatal medical or environmental conditions on PCT levels. In a large series involving 2'151 infants (26 with EONS), Joram et al. identified gestational age (28-32 weeks) and $\mathrm{pH}<7.10$ to be the only factors associated with increased PCT levels ${ }^{48}$. In our series, PCT was strongly correlated with PROM, maternal fever, Apgar score, gestational age, infant heart and respiratory rate, suggesting that prenatal condition and newborn adaptation impact PCT level as displayed by a significant PCT level difference in healthy infants born from either the hospital (with maternal risk factors) or the sub-urban arm (no maternal risk factors). Interestingly, CD74/IP-10 ratio was shown for the first time to have a better accuracy for sepsis diagnosis than any studied biomarkers and clinical combinations. CD74, the invariant chain involved in MHC class II molecules transport, is identified as a prognosis marker in adult critically ill patients developing healthcare associated infections ${ }^{40}$. In our study, CD74 expression alone has a low accuracy for neonatal sepsis diagnosis, but its association with IP-10, the interferon- $\gamma$ inducible protein-10, emerges as a strong combination of biomarker (AUC $0.77,95 \%$ $\mathrm{Cl}: 0.72$ to 0.81$)$. IP-10 is associated with various inflammatory conditions such as autoimmune diseases, hemophagocytic syndromes and viral infections where its combination with phospholipase A2, or IL-10 were suggested for sepsis diagnosis ${ }^{31,52-54}$. Combination of $C D 74$ and IP-10, illustrating both antigen presentation ability and response to type II interferon stimulation, may be representative of both faces of neonatal immunological competency to infection. Ontogeny of antigen presentation in early life is thought to be a key factor in determining age-specific responses to microbes and other antigens. IP-10 production in infants reflects a consequent Th1 response and as such, it may represent in conjunction with a reduced $C D 74$ expression, despite IFNg stimulation, a signature for severely dysregulated response associated with neonatal sepsis.

Although well recognized in cancer, few publications relate $C D 74$ utility in sepsis, none in children and neonates ${ }^{55,56}$. In contrast, in a study of critically ill septic adults, low expression of $C D 74$ was frequently shown to be independently associated with mortality (OR $3.4,95 \% \mathrm{Cl}: 1.2$ to $9.8, \mathrm{p}=0.026$ ) ${ }^{38}$ whereas secondary infection occurrence was associated with increased CD74 expression in the first day following admission for septic shock ${ }^{40}$. Our study further extend $C D 74$ prognosis utility in neonatal sepsis. $C D 74$ expression on cord blood of < 9.47 cutoff value (Youden index 0.09) was predictive of death in neonates with a sensitivity of $95 \%$ and a specificity of $13 \%$. Indirectly those results confirm those related to histocompatibility leukocytes antigen-DR expression on monocytes (mHLA-DR) that is known to be highly 
correlated ( $\mathrm{r}=0.87$ ) with $C D 74$ expression [38]. Low $\mathrm{mHLA}$-DR was shown to be associated with EONS in premature infants and mortality following LONS ${ }^{57,58}$. The underlying pathophysiologic rational behind the association of low CD74/low mHLA-DR in infants and sepsis mortality may be resumed by an altered antigen presentation, either acquired or secondary to a perinatal event. Primary defect or decreased expression of mHLA-DR was shown to be associated with prematurity and altered myeloid cells functionality in extremely immature infants ${ }^{7,59}$. Secondary decrease of mHLA-DR in response to diverse aggression is well recognized. Decrease in mHLA-DR expression was demonstrated following sepsis, and is recognized as one of the main immunological caracterization of post-infective immune failure ${ }^{60-62}$. As such, the prolonged alteration of MHLA-DR was shown to be associated with mortality in adults with sepsis ${ }^{60,63-65}$. Serial mHLA-DR kinetics in septic newborns is largely unknown although a rise in HLA-DR negative monocytes following severe sepsis is reported ${ }^{66}$. Interestingly, in our study, infants surviving sepsis showed a prolonged alteration of $C D 74$ expression up to one month following EONS. Nevertheless, such prolonged post-sepsis reduction in $C D 74$ transcription, as well as reduced mHLA-DR has never been described in both infants and adults. Newborns are known to have a rapidly evolving immunity during the first months of life. Olin et al. demonstrated in a longitudinal analysis of infant's immunity that a phenotypic convergence is occurring between term and preterm infants at three months of age, but in between environmental factors shape immune variation ${ }^{67}$. Interestingly they showed that drastic changes in cell composition and phenotypes, plasma protein concentrations occurred during the first few days of life bringing additional insights to the understanding of biomarkers kinetics and potential utility for diagnosing EONS. Accordingly, our study establishes the reference values of IL-6, IL-10, IP-10 and $C D 74, C X 3 C R 1$ during the first 4 and 12 weeks of life, respectively. These are the first published set of reference values from newborn and infants living in Sub Saharan Africa. Extrapolation of these reference values to infants outside Africa may be acceptable. We showed that gestational malaria did not impact any biomarkers values making these reference values applicable in region with no endemic malaria. In addition, a recent study showed that age-related immune patterns was not different between Africa, North America, South America and Europe despite environmental variations ${ }^{68}$.

Impact of prematurity, as well as perinatal asphyxia are now confirmed as confounding criterion for PCT, and $C D 74$ value interpretation at birth. Prevalence of prematurity in this cohort (31.6\%) is due to the recruitment strategy and may not be representative of Benin epidemiology. Nevertheless, the prevalence of neonatal sepsis in our cohort (29\%) is consistent with previous studies (between $16 \%$ to $28 \%$ ) reducing the likelihood of potential skewness of the studied cohort ${ }^{69,70}$. Our study brings additional epidemiological information about the pathogens encountered in Sub Saharan Africa neonatal sepsis. Beside Staphylococcus spp. predominance as expected in premature infants, specific Enterobacteriacea, such Enterobacter cloacae complex, Klebsiella pneumonia, Serratia marcescens were identified. This certainly raises an important question regarding the presence of resistant Enterobacteriacea usually considered as nosocomial pathogens. The early occurrence of those neonatal sepsis episodes suggests a materno-fetal transmission although hand carried infection could not be excluded. The wide use of over-the-counter antibiotics (eg. Quinolones, blactams) in Africa is known to be associated with 
antimicrobial resistance ${ }^{71}$. All infants, but one, with neonatal sepsis were delivered in University Hospitals for maternal risk factors such as prematurity, PROM, or maternal fever. As per local protocols, use of prepartum antibiotics was performed uniquely if cervical carriage of Streptococcus aglactiae occurred or in febrile mothers, however in practice no mother had any cervical swabs. This observation certainly open significant avenue for infection prevention and strategies focusing at reducing neonatal exposure to resistant pathogens ${ }^{72-74}$.

\section{Conclusion}

In this study cord blood PCT was found to have a low accuracy for neonatal sepsis diagnosis and CD74 to be predictive of mortality in infants with neonatal sepsis. Kinetics of $C D 74$ expression following neonatal sepsis showed a prolonged impairment in survivors. In addition, CD74/IP-10 ratio was found to have a good accuracy for neonatal sepsis diagnosis, depicting a novel signature for defective Th1 response and altered antigen presentation seen in sepsis and sepsis-associated immune depression.

\section{Abbreviations}

CD74, Cluster of differentiation 74; CX3CR1, C-X3-C motif chemokine receptor 1; PCT, Procalcitonin; IL6/-10, Interleukin-6/-10; IP-10, Interferon inducible protein 10; CRP, C reactive protein; ; LMIC, Low- and middle-income country; SSA, sub-saharan Africa; GM, gestational malaria; E-/L-ONS, Early-/late-onset neonatal sepsis; W, week; AUC, Area under the cruve; GA, gestational age; mHLA-DR, monocyte Human leukocyte antigen-DR.

\section{Declarations}

Ethics approval and consent to participate: The study protocol was approved by the local institutional review board (CER-ISBA 85-5). Written informed consent was obtained from parents.

Consent for publication: Not applicable

Availability of data and materials: Data are available upon request to the corresponding author.

Competing interest: JYM, LV, AP are employed by an in vitro diagnostic company, bioMérieux SA. The remaining authors declare that this research was conducted in the absence of any commercial or financial relationship that could cause potential conflict of interest.

Funding: Funded by a consortium including bioMérieux SA, the Institute for Research in Development (IRD), The National Centre for Scientific Research (CNRS), with support of the French National Agency for Research and Technology (ANRT), the Foundation Simone Beer under the auspices of the Foundation of France, and the Foundation for Medical Research (FRM). 
Authors' contribution: GA, SD, VB, MA, MJA, IDD, AM, NF enrolled the patients and collected clinical data; SE, FB performed the statistical analysis; SE, MM, FB, GA, ULT, NF, PT analyzed results and made the figures; NF, VB, LV, JYM, AP, PT designed the research; SE, NF, PT wrote the paper; All authors critically reviewed the manuscript.

Acknowledgements: A list of collaborators in the SEPSIS study group is provided in the Supplementary Appendix 1

\section{References}

1. IHME. Global Burden of Disease Collaborative Network. 2018.

2. Watkins R.R., et al. A Multicenter Survey of House Staff Knowledge About Sepsis and the "Surviving Sepsis Campaign Guidelines for Management of Severe Sepsis and Septic Shock". J Intensive Care Med. 35:187-190 (2020).

3. Bailey P.E., et al. Institutional maternal and perinatal deaths: a review of 40 low and middle income countries. BMC Pregnancy Childbirth.17:295 (2017).

4. Simon A.K., et al. Evolution of the immune system in humans from infancy to old age. Proc Biol Sci.282:20143085 (2015).

5. Glaser K., \& Speer C.P. Toll-like receptor signaling in neonatal sepsis and inflammation: a matter of orchestration and conditioning. Expert Rev Clin Immunol.9:1239-1252 (2013).

6. Collins A., et al. Why are preterm newborns at increased risk of infection? Arch Dis Child Fetal Neonatal Ed.103:F391-F4 (2018).

7. Tissieres P., et al. Innate immune deficiency of extremely premature neonates can be reversed by interferon-gamma. PLoS One.7:e32863 (2012).

8. Medugu N., et al. Aetiology of neonatal sepsis in Nigeria, and relevance of Group b streptococcus: A systematic review. PLoS One.13:e0200350 (2018).

9. Odorizzi P.M., \& Feeney M.E. Impact of In Utero Exposure to Malaria on Fetal T Cell Immunity. Trends Mol Med.22:877-888 (2016).

10. Adatara P., et al. Risk Factors Associated with Neonatal Sepsis: A Case Study at a Specialist Hospital in Ghana. Scientific World Journal.2019:9369051 (2019).

11. Camacho-Gonzalez A., et al. Neonatal infectious diseases: evaluation of neonatal sepsis. Pediatr Clin North Am.60:367-389 (2013). 
12. Ndibazza J., et al. Associations between maternal helminth and malaria infections in pregnancy and clinical malaria in the offspring: a birth cohort in entebbe, Uganda. J Infect Dis.208:2007-2016 (2013).

13. Mpairwe H., et al.. Pregnancy and helminth infections. Parasite Immunol.36:328-337 (2014).

14. Accrombessi M., et al. Effects of Malaria in the First Trimester of Pregnancy on Poor Maternal and Birth Outcomes in Benin. Clin Infect Dis. 69(8):1385-1393 (2019).

15. Le Hesran J.Y., et al. Maternal placental infection with Plasmodium falciparum and malaria morbidity during the first 2 years of life. Am J Epidemiol.146:826-831 (1997).

16. Sylvester B., et al. Prenatal exposure to Plasmodium falciparum increases frequency and shortens time from birth to first clinical malaria episodes during the first two years of life: prospective birth cohort study. Malar J. 15:379 (2016).

17. Labeaud A.D., et al. Do antenatal parasite infections devalue childhood vaccination? PLoS Neg/ Trop Dis. 3:e442 (2009).

18. Savas E.M., et al. Apoptosis Inhibitor of Macrophage, Monocyte Chemotactic Protein-1, and CReactive Protein Levels Are Increased in Patients with Metabolic Syndrome: A Pilot Study. Metab Syndr Relat Disord. 18(4):197-205 (2020).

19. Tzialla C., et al. New Diagnostic Possibilities for Neonatal Sepsis. Am J Perinatol. 35:575-577 (2018).

20. Meem M., et al. Biomarkers for diagnosis of neonatal infections: A systematic analysis of their potential as a point-of-care diagnostics. J Glob Health. 1:201-209 (2011).

21. Benitz W.E., et al. Serial serum C-reactive protein levels in the diagnosis of neonatal infection. Pediatrics.102:E41 (1998).

22. Quadir A.F. \& Britton P.N. Procalcitonin and C-reactive protein as biomarkers for neonatal bacterial infection. J Paediatr Child Health. 54:695-9 (2018).

23. Hornik C.P., et al. Use of the complete blood cell count in early-onset neonatal sepsis. Pediatr Infect Dis J. 31:799-802 (2012).

24. Elbirt D., et al. [Procalcitonin--a specific marker for severe bacterial infection and sepsis]. Harefuah. 140:343-346 (2001).

25. Alima Yanda A.N., et al. [Serum procalcitonin: a reliable tool for the diagnosis and follow-up of neonatal bacterial infections in Sub-Saharan Africa]. Arch Pediatr. 22:1015-1020 (2015).

26. Stocker M., et al. Procalcitonin-guided decision making for duration of antibiotic therapy in neonates with suspected early-onset sepsis: a multicentre, randomised controlled trial (NeoPIns). Lancet. 390:871881 (2017). 
27. Lee J., et al.. The influencing factors on procalcitonin values in newborns with noninfectious conditions during the first week of life. Korean J Pediatr.60:10-16 (2017).

28. Shahkar L., et al. The role of IL-6 for predicting neonatal sepsis: a systematic review and metaanalysis. Iran J Pediatr. 21:411-417 (2011).

29. Ng P.C., et al. IP-10 is an early diagnostic marker for identification of late-onset bacterial infection in preterm infants. Pediatr Res. 61:93-98 (2007).

30. Wagner T.A., et al. Emerging biomarkers for the diagnosis of severe neonatal infections applicable to low resource settings. J Glob Health.1:210-23 (2011).

31. Chen H.L., et al. Plasma IP-10 as a predictor of serious bacterial infection in infants less than 4 months of age. J Trop Pediatr. 57:145-151 (2011).

32. Machado J.R., et al. Neonatal sepsis and inflammatory mediators. Mediators Inflamm. 2014:2696812014.

33. Wu H.P., et al. Serial cytokine levels in patients with severe sepsis. Inflamm Res. 58:385-393 (2009).

34. Poggi C. \& Dani C. Sepsis and Oxidative Stress in the Newborn: From Pathogenesis to Novel Therapeutic Targets. Oxid Med Cell Longev. 2018:9390140 (2018).

35. Pachot A., et al. Systemic transcriptional analysis in survivor and non-survivor septic shock patients: a preliminary study. Immunol Lett. 106:63-71 (2006).

36. Tang B.M., et al. Genome-wide transcription profiling of human sepsis: a systematic review. Crit Care. 14:R237 (2010).

37. Friggeri A., et al. Decreased CX3CR1 messenger RNA expression is an independent molecular biomarker of early and late mortality in critically ill patients. Crit Care. 20:204 (2016).

38. Cazalis M.A., et al. Decreased HLA-DR antigen-associated invariant chain (CD74) mRNA expression predicts mortality after septic shock. Crit Care. 17:R287 (2013).

39. Pachot A., et al. Decreased expression of the fractalkine receptor CX3CR1 on circulating monocytes as new feature of sepsis-induced immunosuppression. J Immunol.180:6421-29(2008).

40. Peronnet E., et al. Association between mRNA expression of CD74 and IL10 and risk of ICU-acquired infections: a multicenter cohort study. Intensive Care Med.43:1013-1020 (2017).

41. Teggert A., et al. Biomarkers for Point-of-Care Diagnosis of Sepsis. Micromachines. 11(3):286 (2020).

42. Seale A.C., et al. Maternal and early onset neonatal bacterial sepsis: burden and strategies for prevention in sub-Saharan Africa. Lancet Infect Dis.9(7):428-38 (2009). 
43. Djenontin A., et al. Culicidae diversity, malaria transmission and insecticide resistance alleles in malaria vectors in Ouidah-Kpomasse-Tori district from Benin (West Africa): A pre-intervention study. Parasit Vectors. 3:83 (2010).

44. Fievet N., et al. SEPSIS project: a protocol for studying biomarkers of neonatal sepsis and immune responses of infants in a malaria-endemic region. BMJ Open. 10:e036905 (2020).

45. Kuhn M., et al. Classification and Regression Training. URL https://CRAN.Rproject.org/package=caret. R package version 6.0-78 (2017).

46. Gonzalez I., et al. Visualising associations between paired 'omics' data sets. BioData Min.5:19 (2012).

47. Chiesa C., et al. Early-Onset Neonatal Sepsis: Still Room for Improvement in Procalcitonin Diagnostic Accuracy Studies. Medicine.94:e1230 (2015).

48. Joram N., et al. Umbilical cord blood procalcitonin level in early neonatal infections: a 4-year university hospital cohort study. Eur J Clin Microbiol Infect Dis. 30:1005-13 (2011).

49. Lencot S., et al. A new procalcitonin cord-based algorithm in early-onset neonatal infection: for a change of paradigm. Eur J Clin Microbiol Infect Dis. 33:1229-38 (2014).

50. Altunhan $\mathrm{H}$., et al. Procalcitonin measurement at 24 hours of age may be helpful in the prompt diagnosis of early-onset neonatal sepsis. Int J Infect Dis.15:e854-858 (2011).

51. Frerot A., et al. Cord blood procalcitonin level and early-onset sepsis in extremely preterm infants. Eur J Clin Microbiol Infect Dis. 38:1651-1657 (2019).

52. Mearelli F., et al. Heterogeneous models for an early discrimination between sepsis and non-infective SIRS in medical ward patients: a pilot study. Intern Emerg Med.9:749-757 (2014).

53. Ng P.C., et al. Early prediction of sepsis-induced disseminated intravascular coagulation with interleukin-10, interleukin-6, and RANTES in preterm infants. Clin Chem. 52:1181-1189 (2006).

54. Maruoka H., et al. IP-10/CXCL10 and MIG/CXCL9 as novel markers for the diagnosis of lymphomaassociated hemophagocytic syndrome. Ann Hematol.93:393-401(2014).

55. Wang Z.Q., et al. CD74 and intratumoral immune response in breast cancer. Oncotarget,8:1266412674 (2017).

56. Cheng S.P., et al. CD74 expression and its therapeutic potential in thyroid carcinoma. Endocr Relat Cancer. 22:179-190 (2015).

57. Birle A., et al. Age-related low expression of HLA-DR molecules on monocytes of term and preterm newborns with and without signs of infection. J Perinatol. 23:294-299 (2003). 
58. Genel F, et al. Monocyte HLA-DR expression as predictor of poor outcome in neonates with late onset neonatal sepsis. J Infect.60:224-228 (2010).

59. Palojarvi A., et al. Low monocyte HLA-DR expression as an indicator of immunodepression in very low birth weight infants. Pediatr Res. 73:469-475 (2013).

60. Pachot A., et al. Messenger RNA expression of major histocompatibility complex class II genes in whole blood from septic shock patients. Crit Care Med.33:31-38 (2005).

61. Payen D., et al. Gene profiling in human blood leucocytes during recovery from septic shock. Intensive Care Med. 34:1371-1376 (2008).

62. Fumeaux T. \& Pugin J. Is the measurement of monocytes HLA-DR expression useful in patients with sepsis? Intensive Care Med. 32:1106-11078 (2006).

63. Satoh A., et al. Human leukocyte antigen-DR expression on peripheral monocytes as a predictive marker of sepsis during acute pancreatitis. Pancreas. 25:245-250 (2002).

64. Pfortmueller C.A., et al.. Assessment of immune organ dysfunction in critical illness: utility of innate immune response markers. Intensive Care Med Exp. 5:49 (2017).

65. Sundar K.M. \& Sires M. Sepsis induced immunosuppression: Implications for secondary infections and complications. Indian J Crit Care Med. 17:162-169 (2013)

66. Fotopoulos F.D., et al. The monocyte HLA-DR expression of healthy and infected premature neonates. Pediatric Research. 45:763 (1999).

67. Olin A., et al. Stereotypic Immune System Development in Newborn Children. Cell. 174:1277-92 e14 (2018).

68. Smolen K.K., et al. Pattern recognition receptor-mediated cytokine response in infants across 4 continents. J Allergy Clin Immunol.133:818-26 e4(2014).

69. Ogundare E., et al. Presentation and outcomes of early and late onset neonatal sepsis in a Nigerian Hospital. Afr Health Sci. 19:2390-2399 (2019).

70. Aku F.Y., et al. Bacteriological profile and antibiotic susceptibility pattern of common isolates of neonatal sepsis, Ho Municipality, Ghana-2016. Matern Health Neonatol Perinatol. 4:2 (2018).

71. Tadesse B.T., et al. Antimicrobial resistance in Africa: a systematic review. BMC Infect Dis.; 17(1):616 (2017).

72. Mitgang E.A., et al. Review and mapping of carbapenem-resistant Enterobacteriaceae in Africa: Using diverse data to inform surveillance gaps. Int J Antimicrob Agents 52:372-84 (2018). 
73. Jesumirhewe C., et al. Carbapenemase-Producing Enterobacteriaceae Isolates from Edo State, Nigeria. Antimicrob Agents Chemother.61(8): e00255-17 (2017).

74. Lester R., et al. Prevalence and outcome of bloodstream infections due to third-generation cephalosporin-resistant Enterobacteriaceae in sub-Saharan Africa: a systematic review. $J$ Antimicrob Chemother. 75:492-507 (2020)

\section{Tables}

Table 1. Patient characteristics 


\begin{tabular}{|c|c|c|c|c|c|c|}
\hline & & Suburban & Hospital & Hospital & $\begin{array}{l}\text { All } \\
\text { patients }\end{array}$ & $\begin{array}{l}p- \\
\text { value }\end{array}$ \\
\hline & & $(n=161)$ & $\begin{array}{l}\text { non } \\
\text { sepsis }(n= \\
248)\end{array}$ & $\begin{array}{l}\text { sepsis } \\
(n=172)\end{array}$ & $(n=581)$ & \\
\hline Female n (\%) & & $81(50.3)$ & $129(52)$ & 77 (44.7) & $\begin{array}{l}287 \\
(49.4)\end{array}$ & 0.33 \\
\hline $\begin{array}{l}\text { Birth weight } \\
\text { (grams) }\end{array}$ & & $\begin{array}{l}3006 \\
{[2800-} \\
3205]\end{array}$ & $\begin{array}{l}2700 \\
{[2350-} \\
3100]\end{array}$ & $\begin{array}{l}2600 \\
{[1755-} \\
3100]\end{array}$ & $\begin{array}{l}2816 \\
{[2400-} \\
3150]\end{array}$ & $\dot{0.001}$ \\
\hline \multirow[t]{4}{*}{$\begin{array}{l}\text { Gestational age } \\
\text { (week) }\end{array}$} & & $\begin{array}{l}39.5 \\
{[38.4-} \\
40.3]\end{array}$ & $\begin{array}{l}38.1 \text { [35.3- } \\
39.6]\end{array}$ & $\begin{array}{l}36.1 \\
{[33.3-} \\
39.2]\end{array}$ & $\begin{array}{l}38.4 \\
{[35.5-40]}\end{array}$ & $\dot{0.001}$ \\
\hline & $\leq 37$ weeks $(\%)$ & $10(6.2)$ & $88(35.4)$ & $86(50)$ & $\begin{array}{l}184 \\
(31.6)\end{array}$ & \\
\hline & $\leq 32$ weeks $(\%)$ & $1(0.6)$ & $8(3.2)$ & $24(14)$ & $34(5.8)$ & \\
\hline & $\leq 28$ weeks $(\%)$ & $0(0)$ & $0(0)$ & $1(0.6)$ & $1(0.1)$ & \\
\hline $\begin{array}{l}\text { APGAR score (1 } \\
\text { minute) }\end{array}$ & & 9 [9-9] & 9 [8-9] & 8 [6-9] & 9 [8-9] & $\dot{0.001}$ \\
\hline $\begin{array}{l}\text { APGAR score (5 } \\
\text { minute) }\end{array}$ & & 10 [10-10] & 10 [9-10] & 9 [8-10] & 10 [9-10] & $\dot{0} .001$ \\
\hline $\begin{array}{l}\text { Hemoglobin } \\
\text { (g/dL) }\end{array}$ & & $\begin{array}{l}15[13.4- \\
16.4]\end{array}$ & $\begin{array}{l}14.8[13.1- \\
16.1]\end{array}$ & $\begin{array}{l}14.9 \\
{[13.5-} \\
16.2]\end{array}$ & $\begin{array}{l}14.9 \\
{[13.3-} \\
16.3]\end{array}$ & 0.49 \\
\hline Hospitalization & & $1(0.6)$ & $136(54.8)$ & $\begin{array}{l}171 \\
(99.4)\end{array}$ & 308 (53) & $\hat{0.001}$ \\
\hline $\begin{array}{l}\text { Hospitalization } \\
\text { duration (Day) }\end{array}$ & & $0[0]$ & $3[1-4]$ & 5 [3-10] & $4[2-7]$ & N.C \\
\hline Death & & $1(0.6)$ & $10(4)$ & $38(22.1)$ & $49(8.4)$ & $\dot{0} .001$ \\
\hline $\begin{array}{l}\text { Timing of death } \\
\text { (Day) }\end{array}$ & & $0[0-0]$ & $1[0.2-2]$ & $3[1-8]$ & $2[1-8]$ & N.C \\
\hline Twins & & $4(0.6)$ & $23(4)$ & 18(3.1) & $45(7.7)$ & \\
\hline $\begin{array}{l}\text { Maternal } \\
\text { characteristics }\end{array}$ & & $n=157$ & $n=222$ & $n=152$ & $n=532$ & \\
\hline Gestate & & $3[2-4]$ & $2[1-4]$ & $3[1-4]$ & $3[1-4]$ & 0.005 \\
\hline $\begin{array}{l}\text { Maternal risk } \\
\text { factors }\end{array}$ & & $12(7.4)$ & $248(100)$ & $\begin{array}{l}172 \\
(100)\end{array}$ & $\begin{array}{l}432 \\
(74.3)\end{array}$ & $\dot{0} .001$ \\
\hline & Fever at delivery* & $3(1.8)$ & $56(22.5)$ & $62(36)$ & 108 & $<$ \\
\hline
\end{tabular}




\begin{tabular}{|c|c|c|c|c|c|c|}
\hline & & & & & (20.3) & 0.001 \\
\hline & $\begin{array}{l}\text { Abnormal } \\
\text { amniotic fluid* }\end{array}$ & $0(0)$ & $151(60.9)$ & $81(47.1)$ & $\begin{array}{l}226 \\
(42.5)\end{array}$ & $\dot{0} 001$ \\
\hline & $\begin{array}{l}\text { PROM }(>18 \\
\text { hours)** }\end{array}$ & $0(0)$ & $77(31)$ & $70(40.7)$ & $\begin{array}{l}137 \\
(25.8)\end{array}$ & $\dot{0} .001$ \\
\hline & Chorioamnionitis* & $0(0)$ & $20(8)$ & $27(15.7)$ & $46(8.6)$ & $\dot{0.001}$ \\
\hline $\begin{array}{l}\text { Malaria at } \\
\text { delivery }\end{array}$ & & $6(3.7)$ & $24(9.7)$ & $19(11)$ & $49(8.4)$ & 0.03 \\
\hline IPT & & $160(99.4)$ & $203(81.8)$ & $\begin{array}{l}143 \\
(83.1)\end{array}$ & $506(87)$ & $\dot{0} .001$ \\
\hline
\end{tabular}

* Associated with multiple risk factors

Biomarkers are expressed as median and IQR (inter-quartile range: [Q1-Q3]). Qualitative data are expressed as numbers and frequency and quantitative data are expressed as medians and IQR (interquartile range: [Q1-Q3]). Qualitative variables were compared using the Chi-squared test (or Fisher's exact test for small expected numbers). Anova test (or the Kruskal-Wallis test when distribution was not normal or when homoscedasticity was rejected) was performed to compare groups. A $p$ value $<0.05$ was considered as significant. PROM, Premature Rupture of Membranes; IPT, Intermittent Preventive Treatment of malaria; $A P G A R$, neonatal adaptation score.

\section{Table 2. Microbiology investigation results}




\begin{tabular}{|c|c|c|}
\hline Pathogens & Standard culture & FilmArray ${ }^{\circledR}$ \\
\hline Gram-positive bacteria & $12(20.3)$ & $23(25.3)$ \\
\hline Enterococcus & & $1(1.1)$ \\
\hline Staphylococcus* & $9(15.2)$ & $14(15.4)$ \\
\hline Staphylococcus aureus* & & $5(5.5)$ \\
\hline Streptococcus agalactiae (group B)* & & $1(1.1)$ \\
\hline streptococcus sp* & $3(5.1)$ & $2(2.2)$ \\
\hline Gram-negative bacteria & $31(52.5)$ & $45(49.4)$ \\
\hline Acinetobacter $s p^{\star}$ & $1(1.7)$ & $2(2.2)$ \\
\hline Campylobacter & & $2(2.2)$ \\
\hline Escherichia coli* & $4(6.7)$ & $6(6.6)$ \\
\hline Enterobacter cloacae complex & $11(18.6)$ & $12(13.2)$ \\
\hline Haemophilus influenzae & & $1(1.1)$ \\
\hline Klebsiella pneumoniae* & $6(10.1)$ & $9(10)$ \\
\hline Moraxella sp & $1(1.7)$ & \\
\hline Serratia marcescens* & $8(13.6)$ & $13(14.2)$ \\
\hline Virus & & $23(25.3)$ \\
\hline Norovirus GI/GII & & $1(1.1)$ \\
\hline Rotavirus A & & $1(1.1)$ \\
\hline Coronavirus OC43 & & $2(2.2)$ \\
\hline Detected Human Rhinovirus/Enterovirus & & $12(13.2)$ \\
\hline Respiratory Syncytial Virus & & $7(7.7)$ \\
\hline Other & $16(27.1)$ & \\
\hline Candida non albicans & $1(1.7)$ & \\
\hline Bacteria of different types (probable contamination) & $14(23.7)$ & \\
\hline Bacteria non-identified & $1(1.7)$ & \\
\hline Total & $59(100)$ & $91(100)$ \\
\hline
\end{tabular}

Data are shown as number (\%) of patients 
* Pathogens found in co-infection

Table 3. Cord blood biomarkers level in neonatal sepsis

\begin{tabular}{|c|c|c|c|c|}
\hline Biomarkers & Suburban (Non sepsis) & Hospital (Non sepsis) & Hospital (sepsis) & p-value \\
\hline \multicolumn{5}{|l|}{ Clinical sepsis } \\
\hline$C D 74 *$ & $4.6[3.2-7.2]$ & $5.2[3.1-7.1]$ & $4.7[2-7.5]$ & 0.03 \\
\hline CX3CR1 & $1[0.8-1.6]$ & $0.8[0.5-1.3]$ & $0.8[0.4-1.5]$ & 0.007 \\
\hline IP-10 [pg/ml] & 105 [72.6-223] & 76.8 [49-134.6] & $81.3[56.9-143.1]$ & $<0.001$ \\
\hline IL-10 [pg/ml] & $4.3[2.9-10.2]$ & $4.8[2.9-8]$ & $5.3[3-13.5]$ & 0.05 \\
\hline IL-6 [pg/ml]** & $23.1[4.5-468.5]$ & 7.3 [2.7-118.5] & $29.9[5.6-661.5]$ & 0.002 \\
\hline PCT $[\mathrm{pg} / \mathrm{ml}]]^{\star \star \star}$ & 383 [225.7-543.7] & 458.3 [320.4-716.4] & 506.6 [358.5-987.3] & $<0.001$ \\
\hline \multicolumn{5}{|c|}{ Clinical Adjudication } \\
\hline$C D 74$ & $4.6[3.2-7.2]$ & $5.3[3.3-7.3]$ & $4.6[1.8-6.8]$ & 0.23 \\
\hline CX3CR1 & $1[0.8-1.6]$ & $0.8[0.5-1.4]$ & $0.7[0.4-1.3]$ & 0.015 \\
\hline IP-10 [pg/ml] & 105 [72.6-223] & $79.5[52.2-134.6]$ & 78.5 [49.8-142.8] & $<0.001$ \\
\hline IL-10 [pg/ml] & 4.3 [2.9-10.2] & $4.7[2.8-7.7]$ & $5.5[3.2-14]$ & 0.30 \\
\hline IL-6 [pg/ml] ${ }^{\star \star \star ~}$ & $23.1[4.5-468.5]$ & 8.3 [2.8-124.7] & $26.8[4.6-661.5]$ & $<0.001$ \\
\hline РCT [pg/ml] & 383 [225.7-543.7] & 443.8 [304.3-670.2] & $583.5[384.5-1022.7]$ & $<0.001$ \\
\hline \multicolumn{5}{|c|}{ Sepsis pronostic } \\
\hline$C D 74 * \star \star$ & $5.3[1.7-11.4]$ & $0.8[0.2-6.9]$ & $4.6[1.8-20]$ & $<0.001$ \\
\hline CX3CR1* & $0.8[0.5-1.4]$ & $0.7[0.3-1.3]$ & $1[0.8-1.6]$ & 0.007 \\
\hline IP-10 [pg/ml] & 77 [51.6-132.5] & $89.7[52.6-160.6]$ & $105[72.6-214.5]$ & $<0.001$ \\
\hline IL-10 [pg/ml] & $4.8[2.9-9.4]$ & 5.5 [3.8-14.1] & $4.3[3-10.3]$ & 0.28 \\
\hline IL-6 [pg/ml]* & $13.9[4.1-212.7]$ & 155.4 [11.7-1069.1] & 22 [4.6-258.1] & 0.01 \\
\hline PCT [pg/ml]** & 460.7 [335.8-758.7] & 671.5 [425-1126.6] & 383.8 [227.8-557.5] & $<0.001$ \\
\hline
\end{tabular}

Biomarkers are expressed as median and IQR (inter-quartile range: [Q1-Q3]). CD74 and CX3CR1 expression are expressed as relative expression to HPRT1. Anova test was performed to compare biomarkers levels between the groups. A $p$ value $<0.05$ was considered as significant. Student's t-test (or the Mann-Whitney t-test when distribution was not normal or Welch test when homoscedasticity was rejected) were performed to compare hospital arm groups $\left({ }^{\star} p \leq 0.05 ;{ }^{*} p<0.01 ;{ }^{\star \star \star} p<0.001\right)$. 
CD74, HLA class II histocompatibility antigen gamma chain; CX3CR1, CX3C chemokine receptor 1; IP10, Interferon gamma-induced protein 10; $L$, Interleukin.

\section{Figures}

\section{Figure 1}

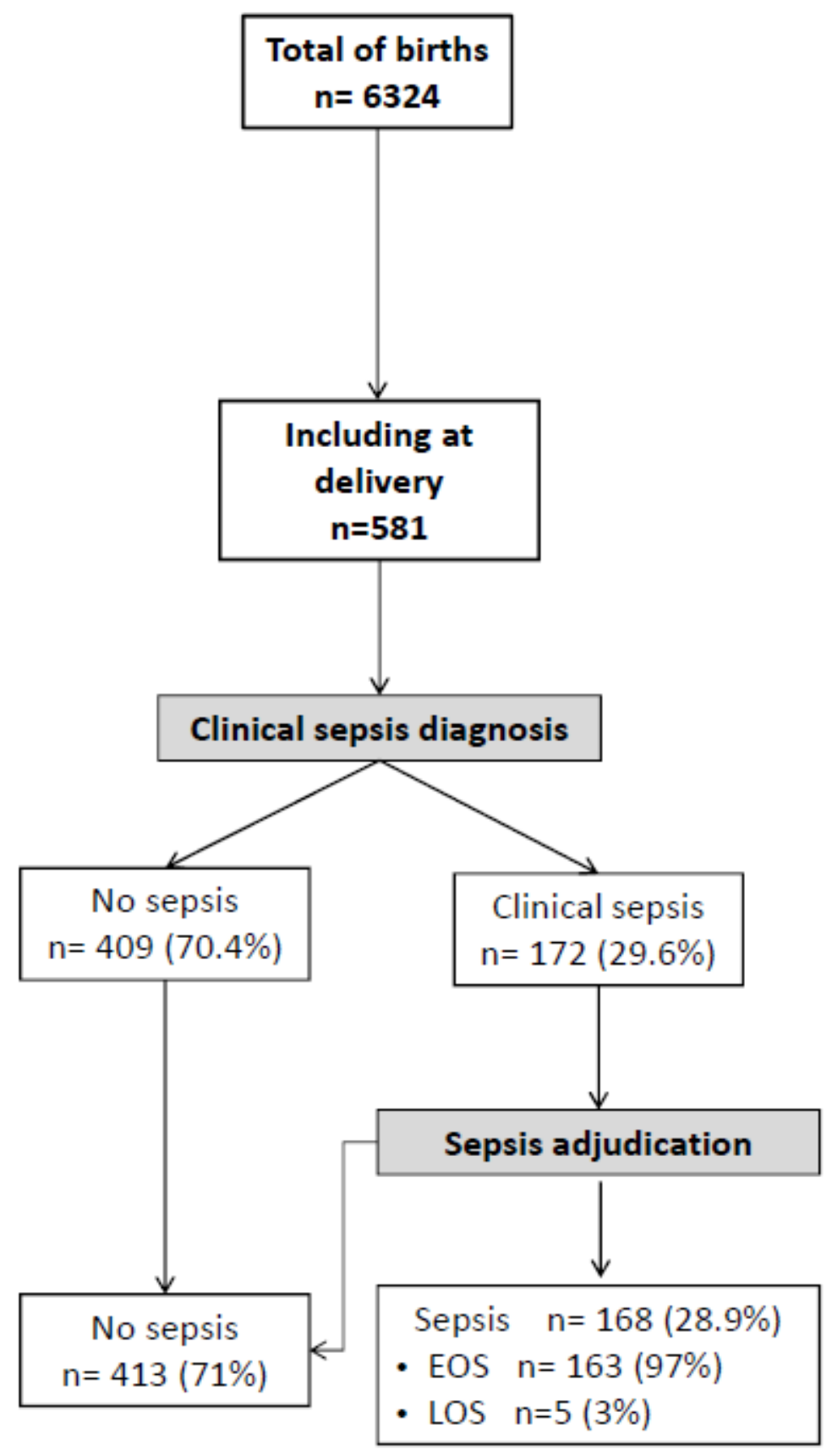

Figure 1 
Flow diagram of study population Recruitment and flow of newborn with risk of neonatal sepsis. At delivery, clinical sepsis diagnosis was established by the local pediatricians based on the child clinical examination and initial workup including haemogram, C-reactive protein (CRP) and microbiological cultures (blood, cerebral fluids and urine). All newborns with a clinical sepsis were subsequently adjudicated by two independent pediatricians and sorted into 'LOS' and 'EOS'.

Figure 2

A
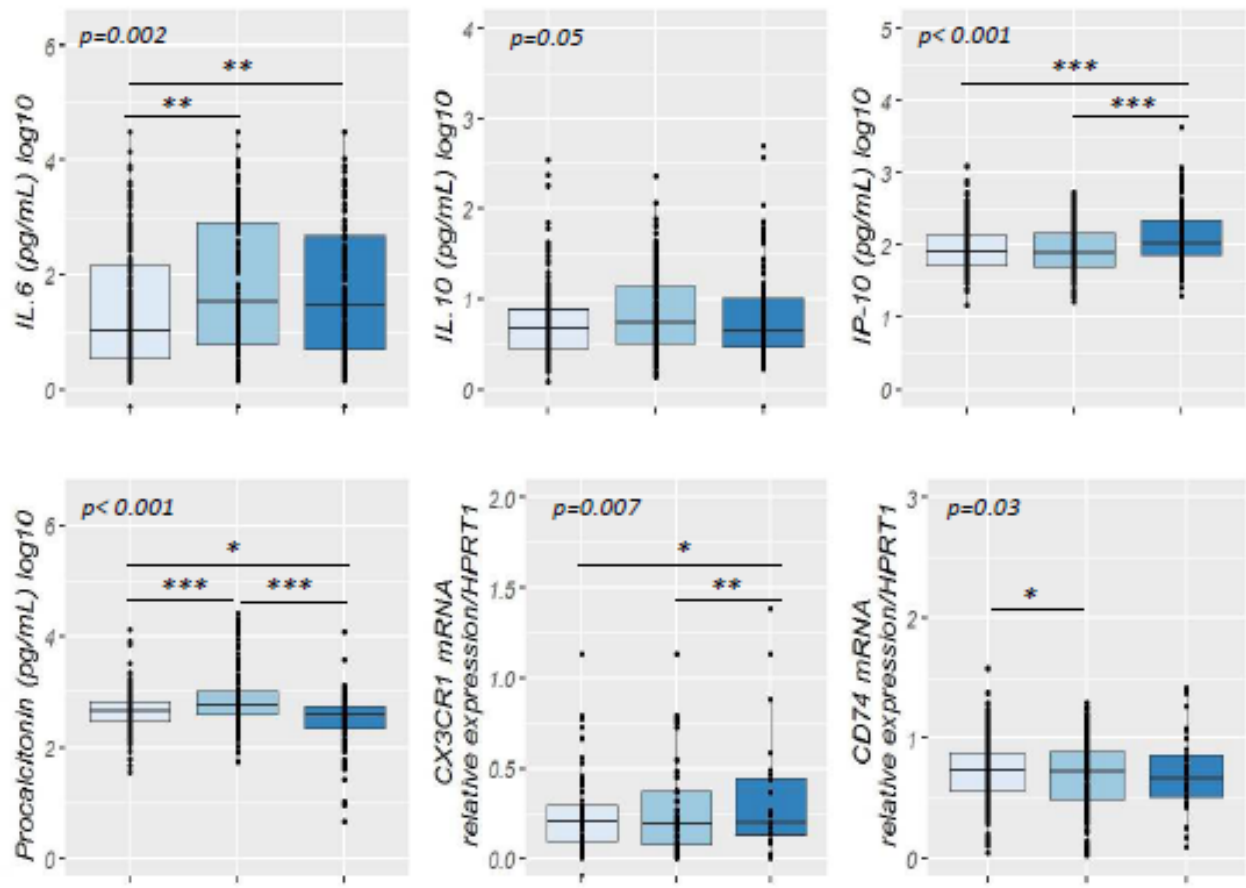

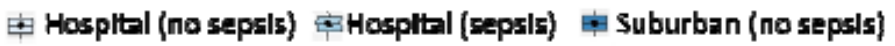

B

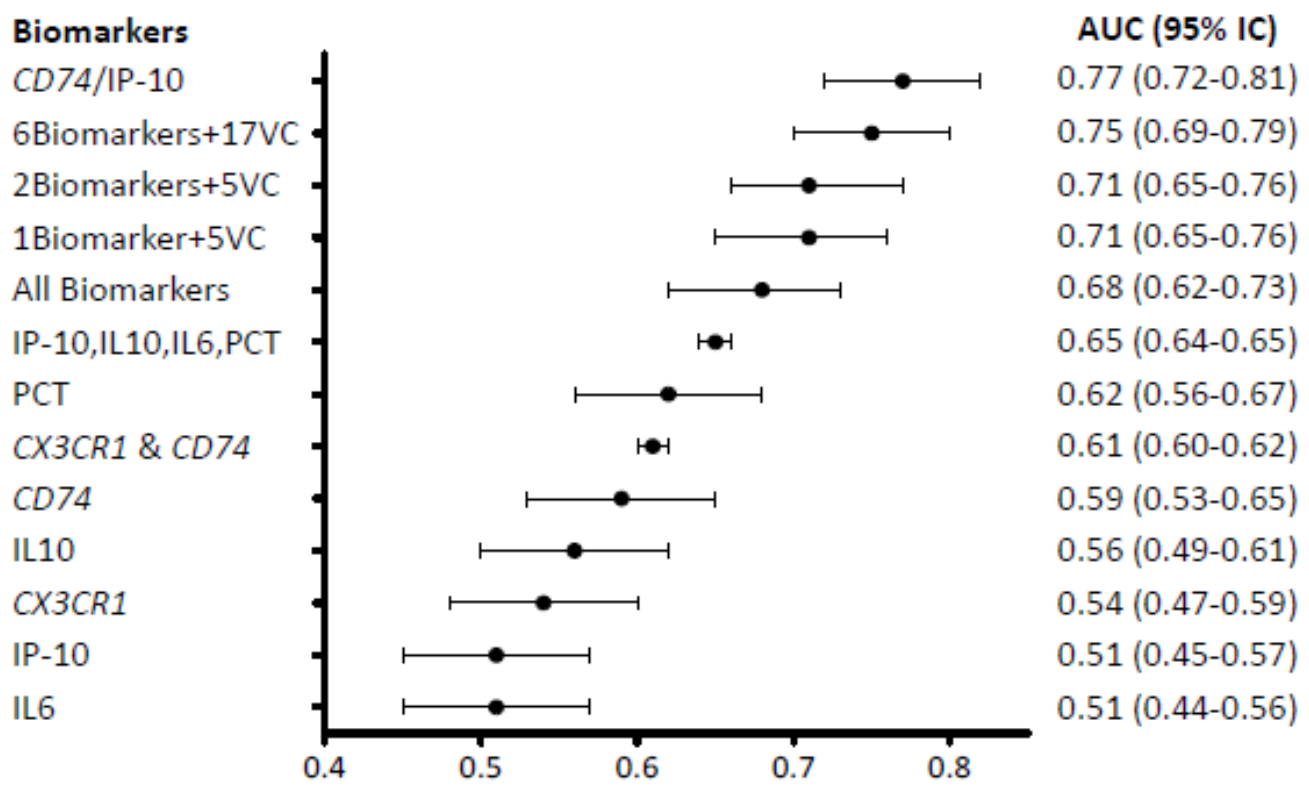

Figure 2 
Cord blood biomarkers level in septic neonates A) Box-plot of transcriptional and protein biomarkers in clinical sepsis diagnosis. CD74 and CX3CR1 mRNA level were evaluated by RT-qPCR with ABI7500 fast and plasma PCT; IL6; IL10 and IP-10 concentrations were measured by multiplexed assay with the Ella platform in cord blood sample obtained from sepsis neonates and no sepsis neonates. Results are presented as box-plots as well as individual values in groups (hospital non-sepsis, hospital sepsis and non-sepsis suburban). Anova test (or the Kruskal-Wallis test when distribution was not normal or when homoscedasticity was rejected) was used to compare biomarkers. A p value $<0.05$ was considered as significant. A post hoc Student $t$ test was performed to compare the groups against each other $\left({ }^{\star} \mathrm{p} \leq 0.05\right.$; $\left.{ }^{* \star} p<0.01 ;{ }^{* \star *} p<0.001\right)$. B) The AUC forest plot of biomarkers used alone, in combination with other biomarkers or in combination with clinical variables to discriminate sepsis and non-sepsis neonates. The AUC presented are those chosen by statistical methods to have cutoffs with 0.95 sensitivity and maximum specificity. The AUC are represented as median and range (95\%IC). 6 biomarkers +17VC (CD74; CX3CR1; IP-10; IL10; IL6; a; b; c; d; e; f; g; h; i; j; k; l; m; n; o; p; q); 2 biomarkers +5VC: IP-10; Procalcitonin; a; h; k; i; q); 1 biomarkers +5VC: Procalcitonin; f; a; h; l; q). Cutoffs value: CX3CR1 (13.55); CD74 (12.24); IL10 $(2.70 \mathrm{pg} / \mathrm{ml}) ; \mathrm{lL} 6(1.44 \mathrm{pg} / \mathrm{ml}) ; \mathrm{IP}-10$ (416.97 pg/ml); PCT (401.81 pg/ml. See clinical variables legend in Supplementary Table 1. 
Figure 3

A

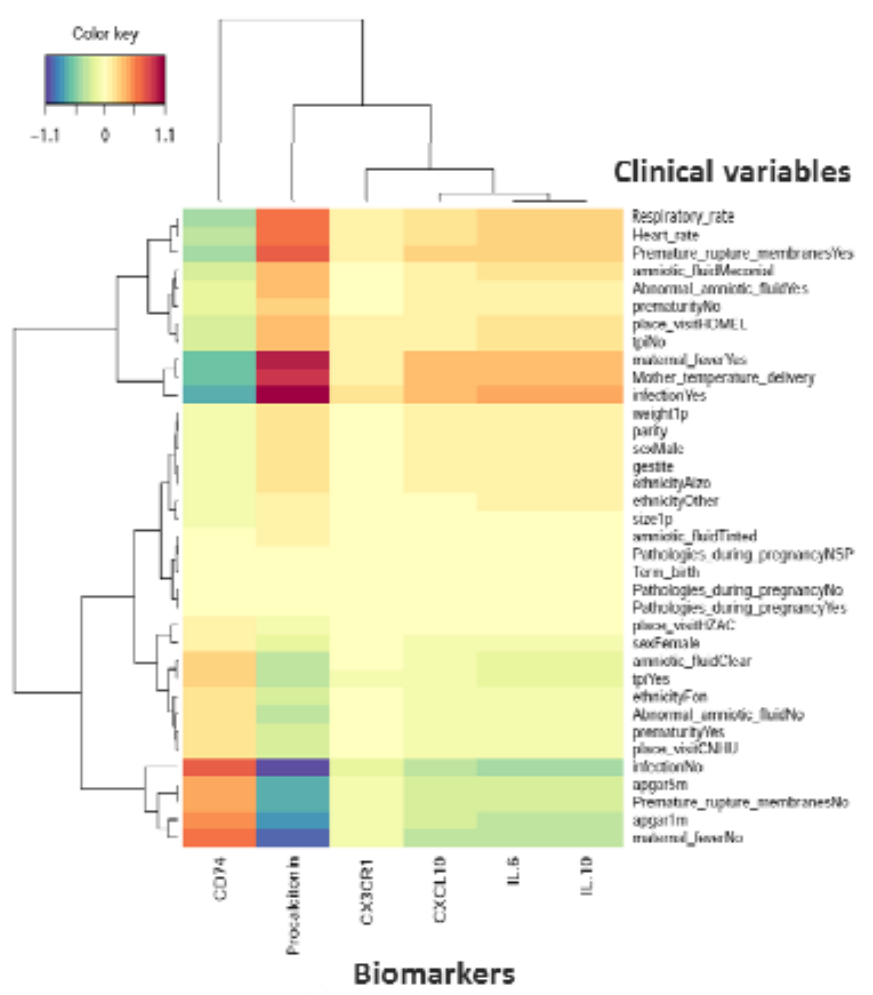

B

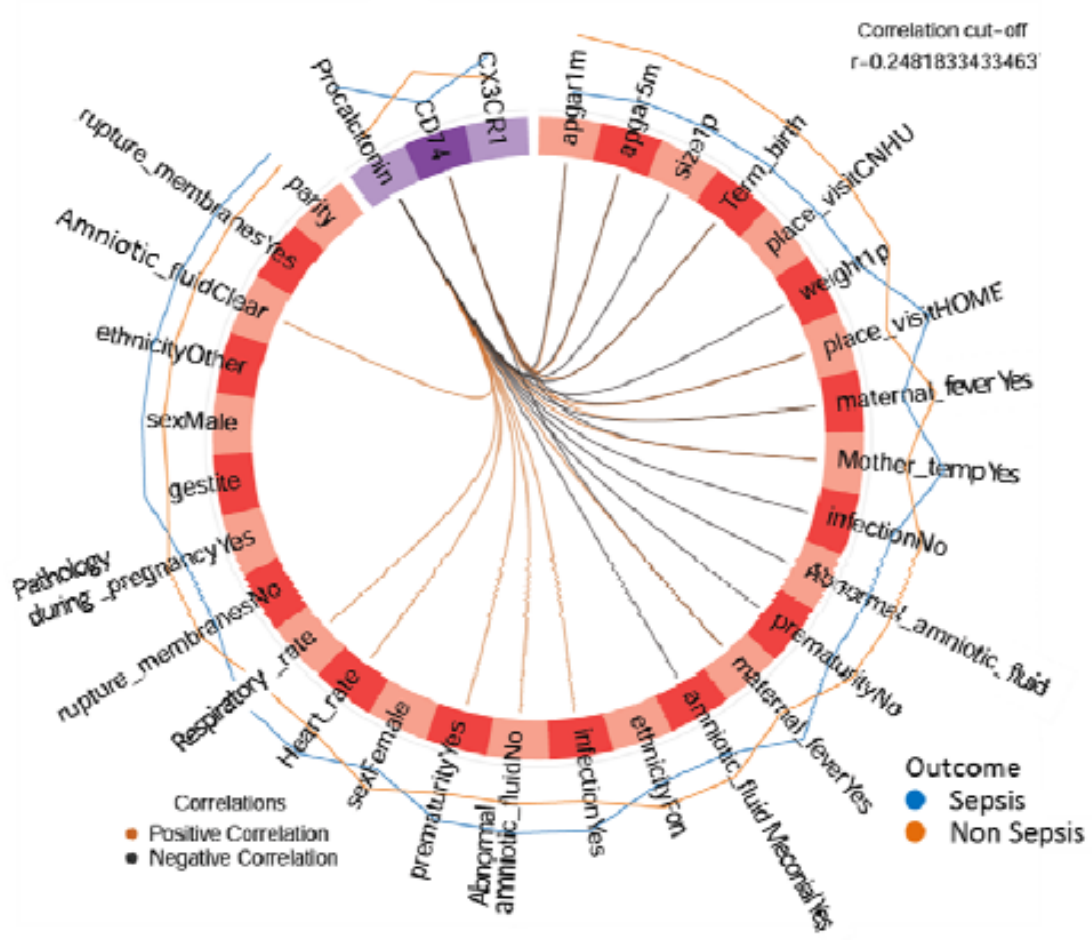

\section{Figure 3}

Biomarkers and clinical variables correlation in neonatal clinical sepsis diagnosis A) Heatmap of correlation matrix (Partial Last Squares Regression) to predict biomarkers from Clinical Variables. The rows and columns correspond to clinical variables and biomarkers. Respectively positive correlations are red and negative correlations are blue. The figure shows only variables with covariances greater than $\max ($ covariance)/2. B) Circosplots of correlations between the same types and different types of 
variables (biomarkers and clinical variables). Positive correlations are shown in orange and negative in black (threshold $r=0.2$ ). The blue and orange lines outside the circle represent the high and low expression levels of each variable respectively according to sepsis and non-sepsis.

Figure 4

A
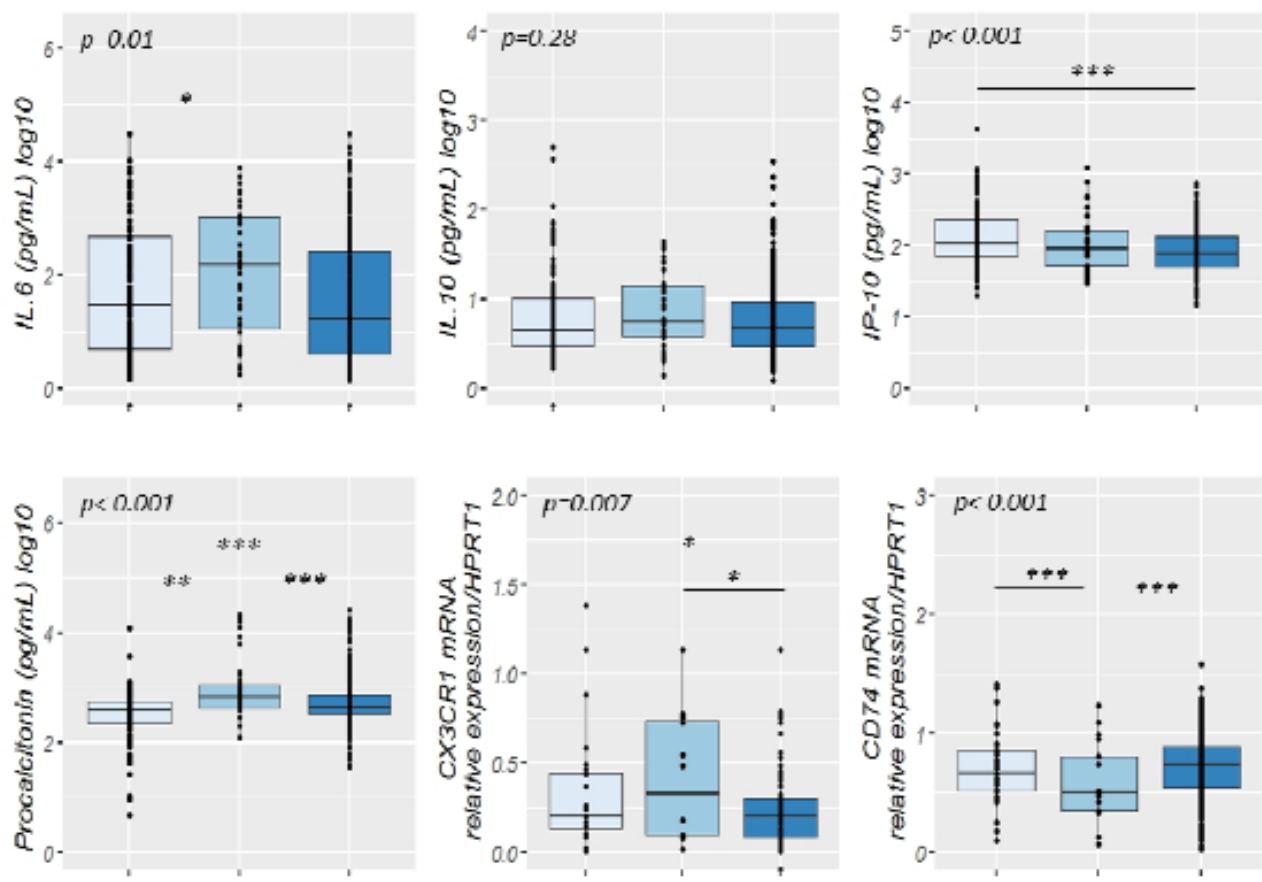

\& Hospital (survivors) $\pitchfork$ Hospital (no survivors) $\$$ Suburban (survivors)

B

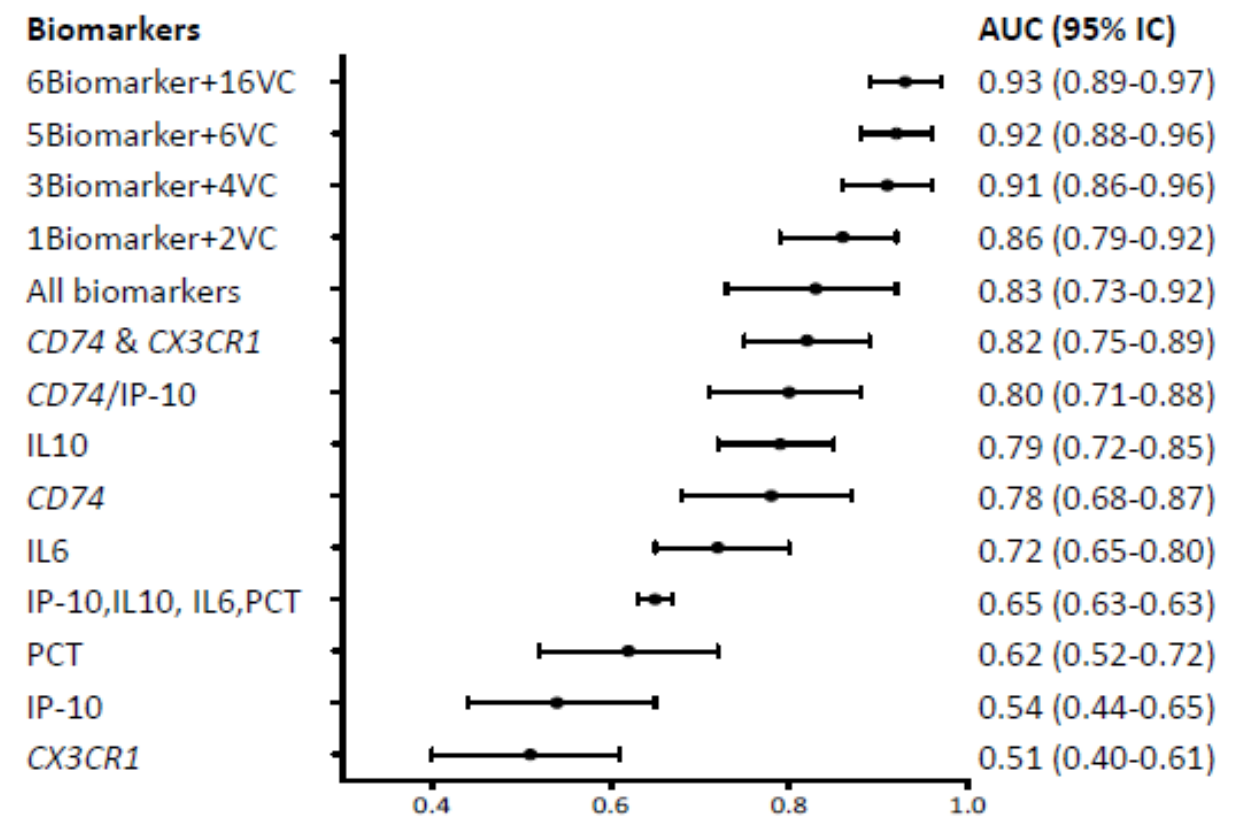

Figure 4

Cord blood biomarkers level according to neonatal sepsis prognostic A) Transcriptional and protein biomarkers expression in sepsis non survivors neonates and survivors neonates. CD74 and CX3CR1 
mRNA level were evaluated by RT-qPCR with ABI7500 fast and plasma PCT; IL6; IL10 and IP-10 concentrations were measured by multiplexed assay with the Ella platform in cord blood sample obtained from non-surviving neonates and surviving neonates. Results are presented as box-plots as well as individual values in groups (hospital survivors, hospital non-survivors and suburban survivors). Anova test (or the Kruskal-Wallis test when distribution was not normal or when homoscedasticity was rejected) was used to compare biomarkers levels in suburban survivors, hospital non survivors and hospital survivor groups (statistically significant test $p \leq 0.05$ ). A post hoc Student $t$ test was performed to compare the groups against each other ( $\left.{ }^{\star} p \leq 0.05 ;{ }^{* *} p<0.01 ;{ }^{* \star *} p<0.001\right)$. B) The AUC forest plot of biomarkers used alone, in combination with other biomarkers or in combination with clinical variables to discriminate sepsis-non surviving neonates and surviving neonates. The AUC presented are those chosen by statistical methods to have cutoffs with 0.95 sensitivity and maximum specificity. 6 biomarkers +16VC (CD74; CX3CR1; IP-10; IL10; IL6; Procalcitonin; s; t; a; b; u; v; f; g; w; j; n; k; l; q; m; r); 5 biomarkers +6VC (Procalcitonin; a; b; h; l; j); 3 biomarkers +4VC (CD74; IP-10; IL 10; a; l; j; m). 1 biomarkers +2VC: (CD74; a; j).Calculated Cutoff : CX3CR1 (1.71); CD74 (9.47); IL-10 (3.75 pg/ml); IL-6 (22.37 pg/ml); IP-10 (44.29 pg/ml); PCT (214.83 pg/ml). See clinical variables legend in Supplementary Table 1. 
Figure 5

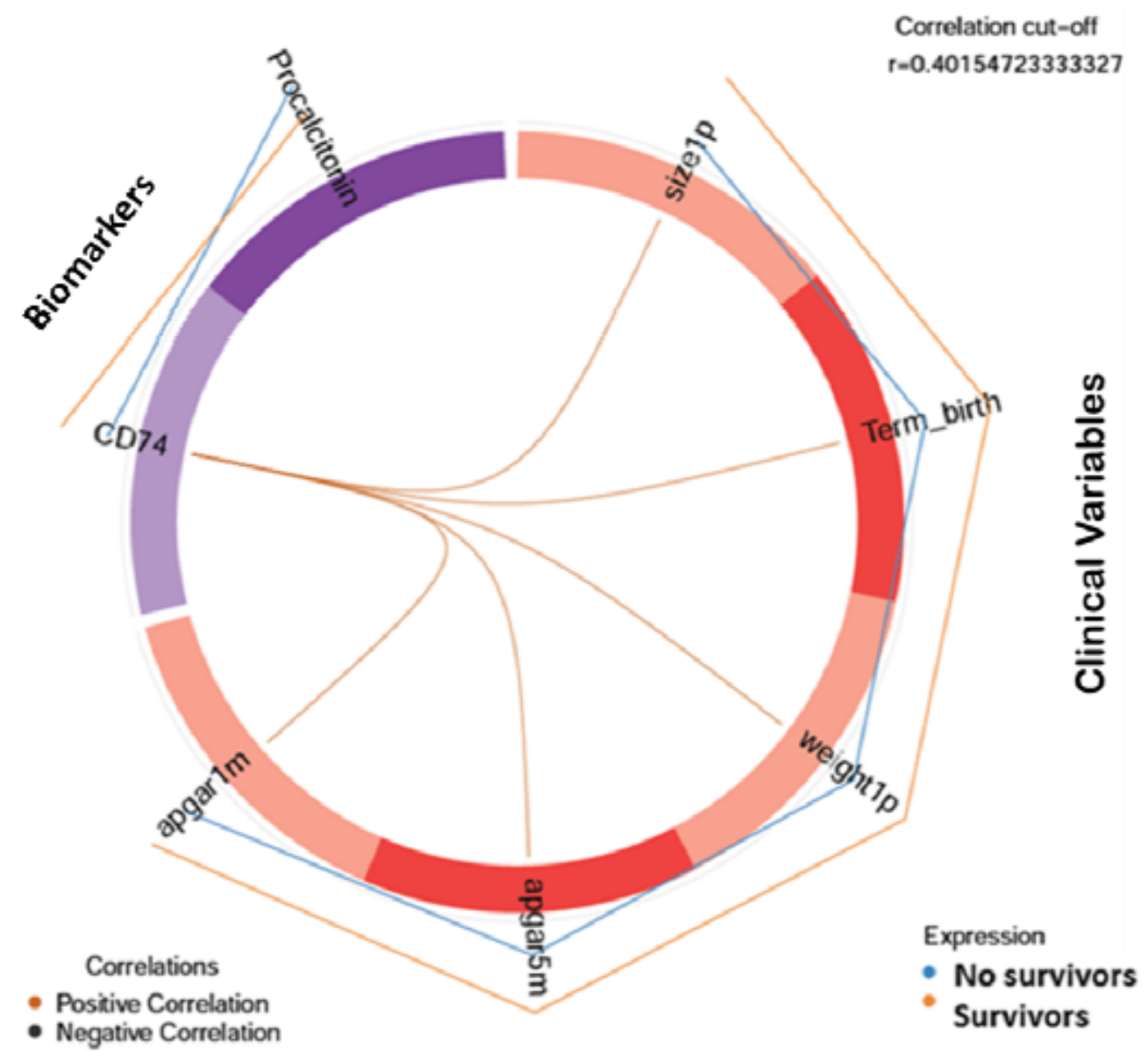

Figure 5

Biomarkers and clinical variables correlation in sepsis neonatal prognostic Circosplots of correlations (PLS Discriminant Analysis) between the same types and different types of variables (biomarkers and clinical variables). Positive correlations are shown in orange and negative in black (threshold $r=0.4$ ). The blue and orange lines outside the circle represent the high and low expression levels of each variable respectively according to survival and death. 
Figure 6
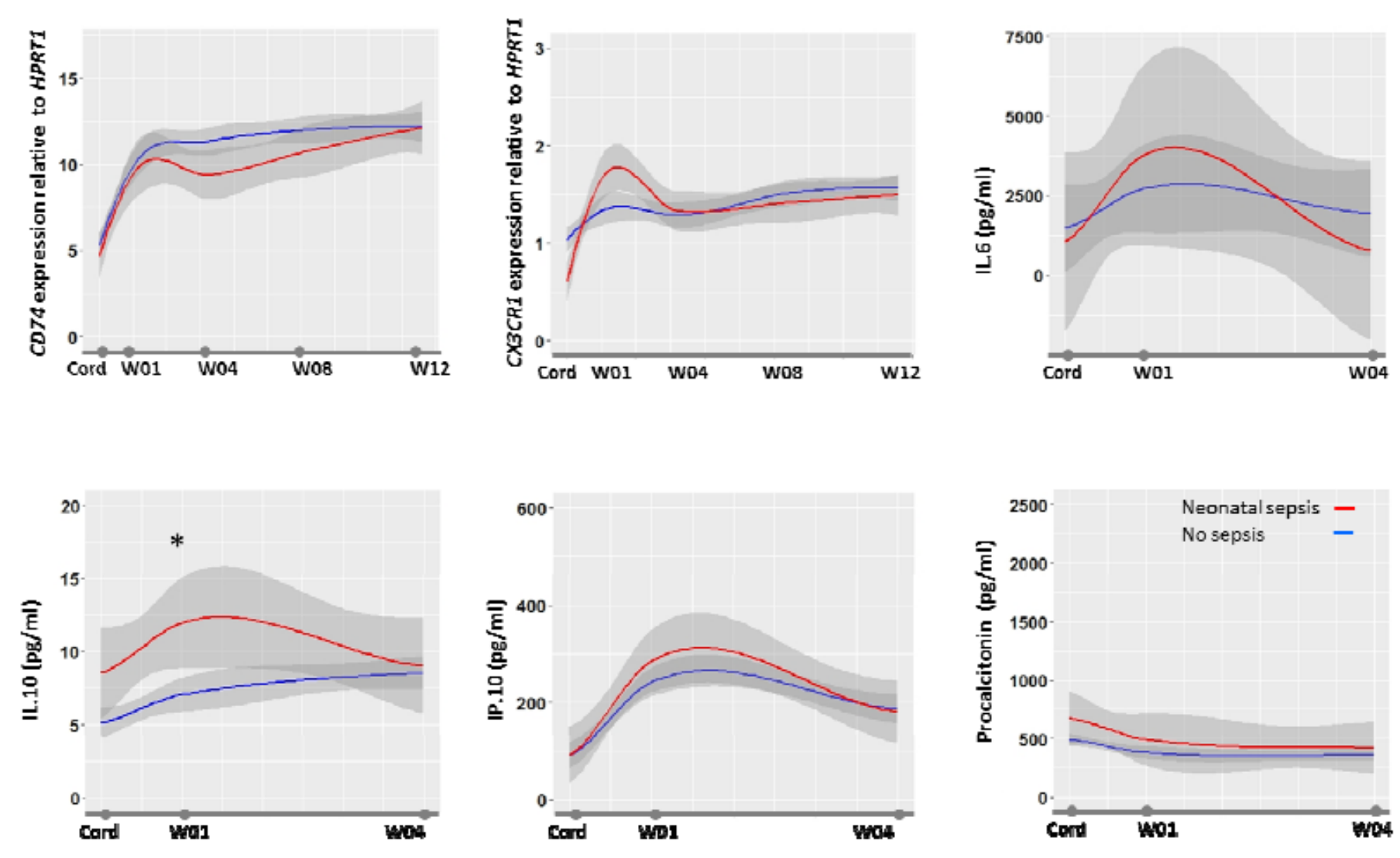

\section{Figure 6}

Biomarkers kinetics during 3 months of follow-up The figure shows the biomarkers profile during the first 3 months of life in healthy infants with median value (in blue), 95\%confidence interval (in gray), and median value in septic infants (in red). Biomarkers were measured at delivery in cord blood (Cord) and at first, four, eight and twelve weeks in peripheral blood. Data are expressed as medians. Anova test was used to compare biomarkers levels.

\section{Supplementary Files}

This is a list of supplementary files associated with this preprint. Click to download.

- SupplementaryAppendix1and2v2.pdf 